\title{
Metástasis gastrointestinales secundarias a adenocarcinoma invasivo pulmonar en un paciente joven: a propósito de un caso
}

\section{Gastrointestinal metastases secondary to aggressive pulmonar adenocarcinoma in a young patient: case report}

\author{
Gabriela Zurita-Aguirre,* Natalia Naveda-Pacheco, ${ }^{*}$ Sergio Poveda-Granja,* Pedro Marín-Castro ${ }^{\ddagger}$ \\ *Hospital «Carlos Andrade Marín», Quito-Ecuador; „Universidad de Especialidades Espíritu Santo, Guayaquil-Ecuador.
}

\begin{abstract}
RESUMEN. El cáncer de pulmón es la principal causa de mortalidad en pacientes oncológicos a nivel mundial. Para que una célula se transforme a cancerígena necesita de varias mutaciones genéticas; dichas mutaciones se producen por múltiples factores predisponentes. La incidencia de este tipo de cáncer es más común en pacientes fumadores y mayores de 40 años; teniendo una aparición menos marcada en pacientes jóvenes. Caso clínico: Presentamos el caso de un paciente masculino de 23 años de edad referido de un hospital de primer nivel por diagnósticos de hemorragia digestiva alta más síndrome constitucional sin llegar a un diagnóstico de base y de difícil manejo. Se realizan exámenes en nuestro nosocomio, los cuales evidencian los hallazgos posteriormente descritos. El paciente tiene una evolución tórpida añadiéndose hemoptisis y signos de insuficiencia respiratoria, negándose a realizar biopsia, llegando a fallecer por múltiples complicaciones; familiares acceden a biopsia posmortem encontrando adenocarcinoma invasivo no mucinoso. Conclusiones: En el presente caso encontramos un paciente con un estadío avanzado de la enfermedad, que presenta un tumor altamente agresivo, como es común en estos casos. La interpretación errónea del cuadro clínico en estadíos iniciales y la falta de interés por parte del paciente para llegar a un diagnóstico conllevaron a un diagnóstico tardío posmortem; aunque desgraciadamente si se lograba un diagnóstico oportuno, el tratamiento oncológico hubiera sido pobre con una baja supervivencia.
\end{abstract}

Palabras clave: Cáncer pulmón, adenocarcinoma pulmón, metástasis gástrica, metástasis duodenal, paciente joven.

\section{INTRODUCCIÓN}

La prevalencia del cáncer de pulmón a nivel mundial es alta y juega un papel muy importante en la mortalidad, siendo la primera causa de muerte por cáncer en el mundo. ${ }^{1}$ Las clases histológicas del cáncer de pulmón son: el carcinoma de células
ABSTRACT. Lung cancer is the type of cancer with the highest mortality in the whole world. For a cell to become cancerous it needs to undergo through several genetic mutations; which occur due to multiple predisposing factors. That is why in this type of cancer it is more common in smokers and patients over 40 years old; having less incidence in young patients. Clinical case: We present a case of a 23-year-old male patient referred from a first-level hospital duo to diagnoses of upper gastrointestinal bleeding + constitutional syndrome that were difficult to manage. Exams were made evidencing described later. Having a torpid evolution adding hemoptysis and signs of respiratory failure, refusing to perform a biopsy, eventually dying from multiple complications; relatives accept a post-mortem biopsy finding invasive non-mucinous adenocarcinoma. Conclusions: In the present case we found a patient in an advanced stage with a highly aggressive tumor as is common in these cases. The erroneous interpretation of the clinical case in the initial stages and the lack of importance on the part of the patient leading to a late diagnosis; although if we make an opportune diagnosis the oncological treatment is poor with a low survival.

Keywords: Lung cancer, lung adenocarcinoma, gastric metastasis, duodenum metastasis, young patient.

pequeñas, carcinoma de células grandes, carcinoma de células escamosas y el adenocarcinoma. ${ }^{2}$ En las etapas más avanzadas se puede desarrollar metástasis a sitios extratorácicos siendo más común al hígado, glándulas suprarrenales, cerebro y huesos. ${ }^{3,4}$ Las metástasis del tracto gastrointestinal son extremadamente raras y presentan una sintomatología variada. ${ }^{5}$

Correspondencia:

Dr. Pedro Marín-Castro

Universidad de Especialidades Espíritu Santo, Guayaquil-Ecuador.

Correo electrónico: pedro.mc91md@gmail.com

Recibido: 10-V-2021; aceptado: 29-X-2021.

Citar como: Zurita-Aguirre G, Naveda-Pacheco N, Poveda-Granja S, Marín-Castro P. Metástasis gastrointestinales secundarias a adenocarcinoma invasivo pulmonar en un paciente joven: a propósito de un caso. Neumol Cir Torax. 2021; 80 (4): 291-295. https://dx.doi.org/10.35366/103454 


\section{PRESENTACIÓN DEL CASO}

Paciente masculino de 23 años de edad, agricultor, nacido y residente de la provincia de Napo, sin antecedentes patológicos personales de importancia, sin antecedentes tabáquicos ni historia familiar de patologías relacionadas. Acude a hospital de primer nivel por rinorrea, alza térmica de predominio nocturno, astenia y pérdida de peso no cuantificada de larga data que se acompaña hace 72 horas con dolor abdominal difuso y hematemesis activa, seguida de deposiciones melénicas no cuantificadas, donde se transfunden tres concentrados globulares y ante no poder controlar el cuadro deciden referir a nuestro hospital. Se realizan estudios complementarios entre estos se encuentran: anemia marcada (hemoglobina $6.7 \mathrm{mg} / \mathrm{dL}$; hematocrito $21.4 \%$ ), marcadores tumorales negativos, excepto CA $125(147.70 \mathrm{U} / \mathrm{mL})$, endoscopia digestiva alta en la que se observa úlcera gástrica y duodenal Forrest III sin toma de biopsia correspondiente, como hallazgo incidental se encuentra Ascaris lumbricoides a nivel del bulbo duodenal; en la radiografía de tórax se observa patrón nodular algodonoso bilateral de predominio en bases (Figura 1); en la tomografía axial computarizada (TAC) de abdomen-pelvis simple se observa hepatoesplenomegalia; en la TAC de tórax simple se contemplan múltiples nódulos con infiltrado de aspecto algodonoso bilaterales, que predominan en las bases donde tienden a confluir, sin signos de necrosis, con derrame pleural basal bilateral y derrame pericárdico leve (Figura 2). Ante estos hallazgos el Servicio de Medicina Interna considera probable síndrome de Löffler, por la IgE francamente elevada, presencia de Ascaris y trastorno pulmonar aparentemente agudo, por lo que inician esquema antiparasitario a base de albendazol, asociado a fluconazol, por hallazgos tomográficos y medidas farmacológicas instauradas, el Servicio de Cirugía Torácica recomienda biopsia pulmonar, la misma que no se realiza por negativa del paciente y familiares.

Posteriormente, se añade tos con expectoración hemoptoica y franca insuficiencia respiratoria, la gasometría arterial reporta alcalosis respiratoria e insuficiencia respiratoria tipo I (pH 7.50, $\mathrm{pCO}_{2} 23.3, \mathrm{pO}_{2} 37, \mathrm{HCO}_{3} 17.7, \mathrm{SatO}_{2} 73 \%$ ), por lo que el paciente es intubado e ingresa a la Unidad de Cuidados Intensivos (UCI). Durante su hospitalización en este servicio presenta choque séptico con requerimientos altos de parámetros ventilatorios (presión positiva al final de la espiración [PEEP]) y sepsis de foco no filiado, informe de estudio microscópico negativo para células neoplásicas en lavado y cepillado bronquial, con eco testicular normal, con rastreo de tuberculosis negativo (reacción en cadena de la polimerasa [PCR] y bacilos ácido alcohol resistentes [BAAR]); con la sospecha de parásitos adicionales (Toxocara), se decide reiniciar esquema antiparasitario más corticoide, el paciente presenta evolución favorable y permite retiro progresivo de soportes ventilatorios y hemodinámicos, egresa de la $\mathrm{UCI}$ al cumplir cinco días de hospitalización e ingresa al Servicio de Medicina Interna.

En controles tomográficos de tórax subsiguientes se reporta regresión parcial de nódulos pulmonares, en ápices algunos nódulos con cavitaciones, nuevos consolidados con broncograma aéreo en segmento 6 izquierdo y base derecha más reporte de laboratorio de Klebsiella pneumoniae multisensible en cultivo de esputo y Staphylococcus aureus meticilino resistente $4 \times 10$ (4) UFC/mL en cultivo de secreción traqueal, se inicia antibioticoterapia a base de ureidopenicilina y glucopéptido; se plantea por segunda ocasión biopsia pulmonar por cirugía torácica, sin embargo, el paciente presenta nueva recaída, precedida de deposiciones melénicas abundantes, caracterizado por respiración paradojal e hipoxemia, es ingresado por segunda ocasión a la $\mathrm{UCl}$ donde se coloca soporte ventilatorio no invasivo como primera instancia, por mal acoplamiento y perpetuación del fallo se procede a ventilación mecánica invasiva (VMI) en modo controlado por presión y se optimiza esquema de antibiótico a base de carbapenémico y glucopéptido, manteniendo antiparasitario (albendazol) y sumando equinocandina por probable componente micótico. El Servicio de Gastroenterología, por persistencia de melenas y choque, realiza nueva videoendoscopia digestiva alta (VEDA), en la que se reporta úlcera gástrica y duodenal Forrest III tomando tejido para estudio histopatológico con resultado de células neoplási-

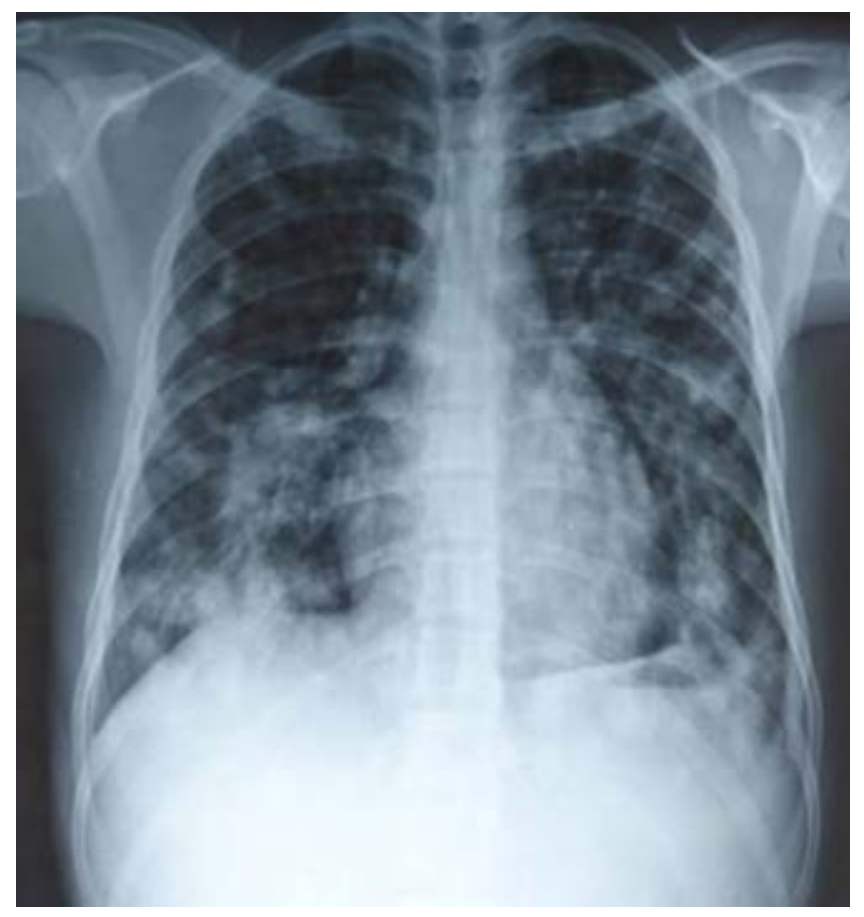

Figura 1: Radiografía de tórax de ingreso. 


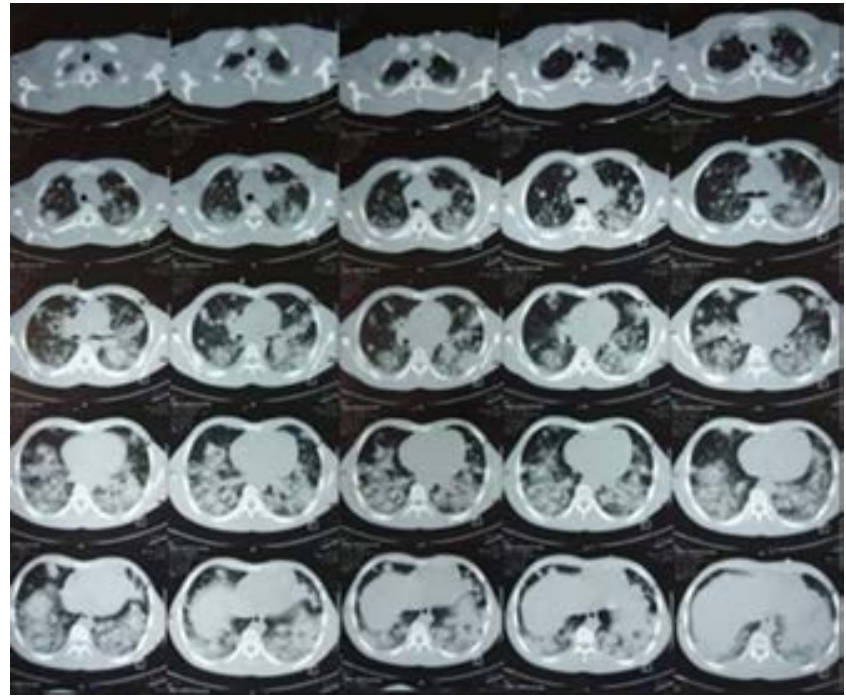

Figura 2: Tomografía computarizada de tórax con hallazgos descritos.

cas pobremente diferenciadas que invaden mucosa (Figura 3), por lo que se considera transfusión de concentrados globulares e infusión de omeprazol, el choque catalogado como mixto hipovolémico y séptico se torna refractario con requerimiento de infusión de hidrocortisona y un segundo vasoactivo. El ecocardiograma reporta derrame pericárdico severo circunferencial con signos iniciales de taponamiento cardíaco, cirugía cardíaca resuelve el cuadro mediante pericardiocentesis, citoquímico compatible con exudado, cultivo del mismo reporta días posteriores $E$. coli multisensible. Paciente con respuesta desfavorable a todas las medidas instauradas, infectología recomienda inicio de itraconazol más anfotericina y antifímicos como última medida de salvataje; el paciente se encuentra con requerimientos altos de parámetros ventilatorios (PEEP) todo el tiempo, desarrolla tolerancia a fármacos sedantes con la complicación subsecuente de fístula broncopleural, finalmente se consigue sedación profunda con remifentanilo y propofol.

En el contexto de un paciente con evolución catastrófica, sangrado digestivo persistente, pancitopenia y necesidad de transfusión de hemoderivados a repetición, además de fallo multiorgánico en el que destaca el respiratorio y cardiovascular, a pesar de varias estrategias ventilatorias con tendencia a la hipoxemia e hipercapnia que contribuye a la perpetuación de la acidosis de componente mixto, hemodinámicamente inestable con dosis máximas de vasoactivos (norepinefrina y adrenalina) con microdinamia, y después de haber recibido antibioticoterapia de amplio espectro, antifúngicos, antifímicos y antiparasitarios sin respuesta a ninguno de ellos, se consigna orden de no reanimación cardiopulmonar (RCP) y limitación del esfuerzo terapéutico.
El paciente fallece a los 23 días de la segunda hospitalización en la $\mathrm{UCl}$. Cirugía torácica realiza biopsia pulmonar posmortem con informe posterior de adenocarcinoma invasivo no mucinoso en patrón de crecimiento lepídico, neumonitis intersticial y daño alveolar difuso.

\section{DISCUSIÓN}

El cáncer de pulmón en adultos jóvenes es una patología rara y tiene características que lo diferencian de las presentaciones en los adultos mayores, normalmente es de características más agresivas; y es más común encontrarlo en hombres que en mujeres. ${ }^{1}$ El subtipo histológico más frecuente es el adenocarcinoma. ${ }^{2}$ Alrededor de 55\% de los pacientes al momento del diagnóstico ya presentan metástasis a distancia. ${ }^{4}$

Las metástasis que migran del cáncer de pulmón al tracto gastrointestinal son raras; siendo más común encontrarlas en el hígado, las glándulas suprarrenales, huesos y cerebro. ${ }^{5}$ El lugar más frecuente de las metástasis gastrointestinales es el intestino delgado, con mayor frecuencia en el yeyuno, íleo y duodeno; siguiendo las metástasis gástricas y por último las colónicas. ${ }^{6,7}$ El adenocarcinoma pulmonar es el tipo histológico más frecuente encontrado en las metástasis gástricas y del intestino delgado. ${ }^{8}$

La vía de implantación de las metástasis es por vía hematógena; las células migran hasta la submucosa gástrica; es por eso que sólo se llega a un diagnóstico cuando hay un crecimiento considerable de la metástasis. ${ }^{4}$ Los pacientes con cáncer de pulmón primario con metástasis al tracto digestivo normalmente son asintomáticos al ini-

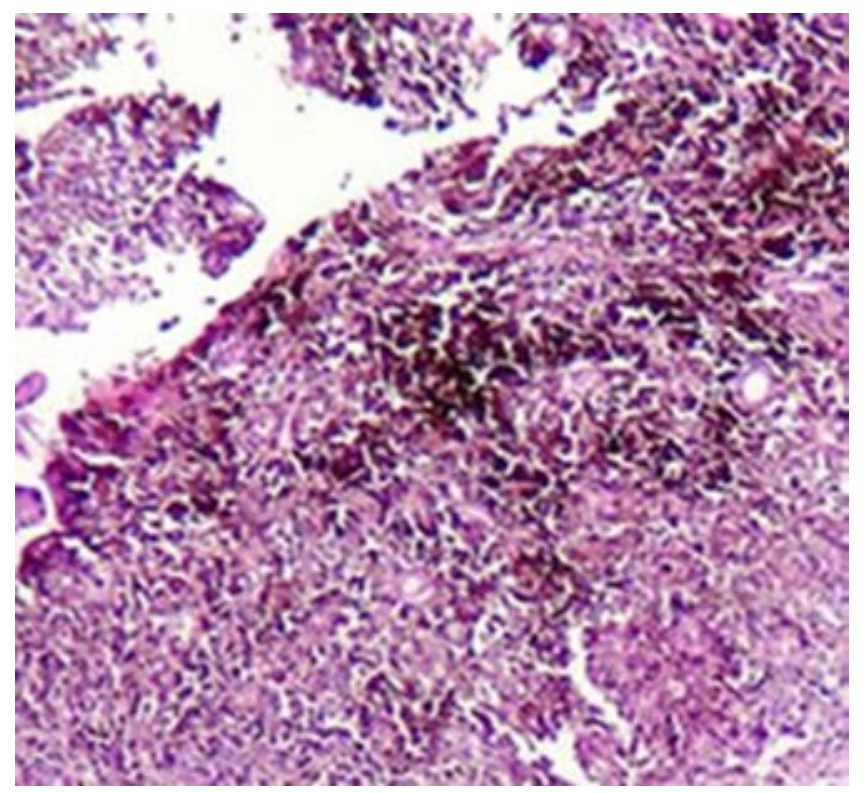

Figura 3: Estudio de histopatología de metástasis gástrica. 
cio. ${ }^{8,9}$ Llegan a producir síntomas cuando ya han invadido la totalidad de la pared gástrica o intestinal; es un tumor de crecimiento rápido que ocluye la luz endoluminal, pudiendo desarrollar síntomas de obstrucción intestinal, aunque es más común encontrar que debuten como una perforación de víscera hueca por la necrosis central que se produce. ${ }^{10-12}$

El sangrado digestivo producto de una metástasis de un cáncer de pulmón es muy raro. ${ }^{4-6}$ Las manifestaciones clínicas más frecuentes cuando hay compromiso del tracto gastrointestinal suelen ser dolor abdominal crónico en el epigastrio y sangrado crónico debido a melena y anemia. . $-6,13^{-13}$

En nuestro caso presentamos un paciente que aparentemente se encontraba sano, sin diagnóstico ni historia personal o familiar de cáncer; debutando su patología con sangrado digestivo y síndrome constitucional. El diagnóstico de metástasis gastrointestinal fue sospechado desde la segunda endoscopia y confirmado con los resultados histopatológicos posmortem de la biopsia pulmonar. Es importante considerar la presencia de metástasis gastrointestinales en todo paciente con cáncer de pulmón que presente síntomas como melena, dolor abdominal, dispepsia, distención o que acuda a emergencia con inestabilidad hemodinámica de causa no conocida. También es importante recordar que se debe investigar un sangrado oculto en pacientes con cáncer de pulmón y anemia por deficiencia de hierro o caída de hemoglobina y hematocrito, incluso en pacientes asintomáticos.

El sangrado gastrointestinal debe ser tratado de manera conservadora con fluidos y transfusiones sanguíneas hasta que el paciente se encuentre hemodinámicamente estable y la hemorragia pare. ${ }^{4-6}$ Sin embargo, en casos de sangrado masivo difícil de controlar se requiere plantear una resección quirúrgica para controlar el sangrado; si encontramos un paciente con cáncer metastásico estable, lo ideal sería el manejo con tratamiento de soporte quimioterápico sin cirugía debido a que nos encontramos ante un paciente con un estadío avanzado. ${ }^{14,15}$

La prognosis de las metástasis gástricas y duodenales en los pacientes con cáncer de pulmón primario es muy baja. ${ }^{14,15}$ Después de un episodio de sangrado digestivo la supervivencia se pronostica de semanas a pocos meses. En el paciente presentado la supervivencia fue baja; superando las cinco semanas desde el inicio de los síntomas y llegando a fallecer por múltiples complicaciones relacionadas a su enfermedad base.

\section{CONCLUSIONES}

Las metástasis gastrointestinales son raras y suelen ocurrir en pacientes que presentan una enfermedad terminal, produciendo muy pocos síntomas. El sangrado del tubo digestivo como síntoma de aparición de la enfermedad es muy raro y está altamente relacionado a una prognosis mala. Teniendo en cuenta todo lo leído, el sangrado gastrointestinal en un paciente con cáncer de pulmón debe ser considerado y estudiado como diagnóstico diferencial una probable metástasis.

\section{REFERENCIAS}

1. Cabo A, del Campo E, Rubio T, Nápoles N, Columbie C. Aspectos clínicos y epidemiológicos en pacientes con cáncer de pulmón en un servicio de neumología. MEDISAN [Internet]. 2018;22(4):394405. Disponible en: http://scielo.sld.cu/scielo.php?script=sci_ arttext\&pid=S1029-30192018000400009\&lng=es

2. Romaszko A, Doboszynska A. Mutliple primary lung cancer: a literature review. Adv Clin Exp Med [Internet]. 2018;27(5):725-730. doi: https://doi.org/10.17219/acem/68631.

3. Dones A, Rivera K, Fernandez R, Adorno J, Hernandez R, González $\mathrm{M}$ et al. An aggressive non-small cell lung cancer in nonsmokers: a case report of an unusual presentation of micropapillary lung adenocarcinoma. Respir Med Case Rep [Internet]. 2017;20:125-128. doi: https://doi.org/10.1016/j.rmcr.2017.01.012.

4. Hu Y, Feit N, Huang Y, Xu W, Zheng S, Li X. Gastrointestinal metastasis of primary lung cancer: an analysis of 366 cases. Oncol Lett [Internet]. 2018;15(6):9766-9776. doi: https://doi.org/10.3892/ol.2018.8575.

5. Balla A, Subiela J, Bollo J, Martínez C, Rodríguez C, Hernández P, et al. Metástasis gastrointestinales de carcinoma pulmonar primario. Serie de casos y revisión sistemática de la literatura. Rev Cir Española [Internet]. 2018;96(4):184-197. doi: https://doi.org/10.1016/j. ciresp.2017.12.011.

6. Yen-Min H, Tsan-Yu H, Jim-Ray C, Hui-Ping C, Pei-Hung C, Cheng$\mathrm{Hsu}$ W, et al. Gastric and colonic metastases from primary lung adenocarcinoma: a case report and review of the literature. Oncol Lett [Internet]. 2012;4:517-520. doi: https://doi.org/10.3892/ol.2012.778.

7. Kim MJ, Hong JH, Park ES, Byun JH. Gastric metastasis from primary lung adenocarcinoma mimicking primary gastric cancer. World J Gastrointest Oncol [Internet]. 2015;7(3):12-16. doi: https://doi. org/10.4251/wjgo.v7.i3.12.

8. Li X, Li S, Ma Z, Zhao S, Wang X, Wen D. Multiple gastrointestinal metastases of squamous-cell lung cancer: a case report. Medicine (Baltimore) [Internet]. 2018;97(24):e11027. doi: https://doi.org/10.1097/ MD.0000000000011027.

9. Pararas N, Kirkilessis G, Pikoulis A, Syrigos K, Pikoulis E. A rare case of a metastatic lung squamous cell carcinoma to the large bowel and the liver. Cureus [Internet]. 2021;13(3):e13867. doi: https://doi. org/10.7759/cureus.13867.

10. Parker NA, McBride C, Forge J, Lalich D. Bowel obstruction caused by colonic metastasis of lung adenocarcinoma: a case report and literature review. World J Surg Oncol [Internet]. 2019;17(1):63. doi: https://doi.org/10.1186/s12957-019-1611-y.

11. Wang J, Chen Y, Zhang S, Chen Q. Perforation of small intestine secondary to metastatic lung adenocarcinoma: a case report. Medicine (Baltimore) [Internet]. 2018;97(49):e13469. doi: https://doi. org/10.1097/MD.0000000000013469.

12. Ahmed A, Nasir UM, Delle P, Swantic V, Ahmed S, Lenza C. A rare presentation of poorly differentiated lung carcinoma with duodenal metastasis and literature review. Case Rep Gastroenterol [Internet]. 2020;14(1):186-196. doi: https://doi.org/10.1159/000506927. 
13. Garavello A, Fransvea P, Rossi S, Giacovazzo F, Marino V. Bowel perforation secondary to metastatic lung cancer: Report of two cases with literature review. Int J Surg Case Rep [Internet]. 2018;51:331-334. doi: https://doi.org/10.1016/j.ijscr.2018.07.039.

14. Lee PC, Lo C, Lin MT, Liang JT, Lin BR. Role of surgical intervention in managing gastrointestinal metastases from lung cancer. World J Gastroenterol [Internet]. 2011;17(38):4314-4320. doi: https://doi. org/10.3748/wjg.v17.i38.4314.
15. Blumenthal G, Bumm P, Chaft J, McCoach C, Perez E, Scagliotti V et al. Current status and future perspectives on neoadjuvant therapy in lung cancer. J Thorac Oncol [Internet]. 2018;13(12):1818-1831. doi: https://doi.org/10.1016/j.jtho.2018.09.017.

Conflicto de intereses: Los autores declaran no tener conflicto de intereses. 